\title{
- Balancing costs and benefits of pandemic control in an
}

\section{outbreak phase}

\author{
Tsuyoshi Hondou \\ Graduate School of Science, Tohoku University \\ Sendai 980-8578, Japan
}

After the first lockdowns in response to the COVID-19 outbreak, many countries faced difficulties in balancing infection control with economics. Because there was limited prior knowledge ${ }^{1)}$, economists began researching this issue ${ }^{2,3,4,5,6)}$ using cost-benefit analysis $^{7,8)}$ and found that infection control processes significantly affect economic efficiency. Rowthorn and Maciejowski ${ }^{2,4)}$ used economic parameters in the United Kingdom to numerically demonstrate that an optimal balance was found in the process, including keeping the infected population stationary. However, universally applicable knowledge, which is indispensable for guiding principles of infection control, has not yet been developed because these analyses assume regional parameters and a specific disease. Here, we prove the universal result of economic irreversibility, which means that delaying infection control measures is more expensive than implementing infection control measures early while keeping infected populations stationary. This means that once the infected population increases, society cannot return to its previous state without extra expenditures. This universal result is analytically obtained by focusing on the infection-spreading 
phase of pandemics, which is not only applicable to COVID-19, and whether or not 'herd immunity' exists $^{11,12)}$. It also confirms the numerical observation of stationary infected populations in its optimally efficient process $^{2,4)}$. Our findings suggest that economic irreversibility is a guiding principle for balancing infection control with economic effects.

Governments in several countries fear adverse economic effects and have hesitated to take measures to control COVID-19 infection because the economic effects may result in illness and death in the non-infected population ${ }^{2}$. For example, Japan hesitated to respond to the pandemic. The Japanese government requested that governors increase their medical capacities ${ }^{10)}$ as they determined the upper limit for the infected population. This social turbulence is attributed to insufficient knowledge about the relationship between infection control and the economy.

Several economists, perceiving a serious lack of knowledge ${ }^{1)}$, started studying this issue spring $2020^{3,4,5,6)}$. Rowthorn ${ }^{2)}$, along with his colleague Maciejowski ${ }^{4)}$, utilised the costbenefit analysis $(\mathrm{CBA})^{7,8)}$ to determine how infection control intervention costs could efficiently be utilised (inhibition of infection). Using the susceptible-infected-recovered (SIR) model to simulate the epidemic ${ }^{9)}$, they discussed several infection control processes to determine the optimal process. The optimal process includes the stationary state of the constant infected population in its principal part. These results were obtained using numerical simulation because Rowthorn assumed that explicit solution was unavailable in this issue ${ }^{2)}$. While the methodology and results of this study ${ }^{2,4)}$ are pioneering and significant, they are not straightforward enough to generalise their results because the study investigated specific situations with given parameter sets. Therefore, explicit solutions independent of specific parameters are needed to reveal the universal property. Explicit solutions could be applicable in the United Kingdom and other countries during different situations, including COVID-19 and other pandemics.

From the physics perspective, the optimisation in CBA is similar to finding the minimum state of energy. In addition, the finding ${ }^{2,4)}$ that the most efficient process includes the stationary 
state suggests an analogous structure with thermodynamic irreversibility.

In this paper, we analytically show the basic property of economic cost in the infection control process by analysing the cyclic processes of the system's state variable. For this purpose, we restrict ourselves to the infection-spreading phase in the pandemic model, in which the infected population grows exponentially in the absence of infection control. In several pandemics, including COVID-19, society may not arrive at a traditional immune state called 'herd immunity' as indicated in several studies ${ }^{11,12)}$. However, the infection-spreading phase is universal and principal, irrespective of whether herd immunity exists. By comparing the stationary state of a constant infected population, we will derive several explicit solutions and inequalities of costs in infection control processes and show economic irreversibility in infection control. With these explicit results, we prove that delaying infection control measures is always more expensive than implementing early measures while keeping the infected population stationary.

\section{Formulation with Cost-Benefit Analysis}

Infection control comprises measures taken to decrease the number of people infected by an individual. The average number within society is called the 'effective reproduction number', $R_{t}$. When $R_{t}$ drops below 1, epidemics subside. Several measures, including handwashing, wearing masks, suspension of business activities, and lockdowns can be taken to reduce $R_{t}$ from its uncontrolled (natural) value, $R_{N}>1$. $R_{N}$ equals the basic reproduction number $R_{0}$ for the initial phase of infection. These measures have a negative influence on the economy and society ${ }^{2)}$. This social cost, $\hat{C}$, is positively correlated to the strength of the measure. Rowthorn assumed ${ }^{2)}$ that the infection control measure is taken through the value of $q$ as $R_{t}=R_{N}(1-q(t))$, where $q$ represents the intensity of social intervention against pandemics. Then, he defined the social cost per unit of time as a function of $q$ : $\hat{C}=\hat{C}(q)^{2,4)}$. He assumed $\hat{C}(0)=0$ because there is 
no infection control at $q=0$.

Here, we consider the social cost induced by the infection measure as a function of the effective reproduction number, $R_{t}$, instead of $q$. While Rowthorn ${ }^{2)}$ assumes maximum strength, $q_{\max }$, which corresponds to the minimum effective reproduction number, $R_{t}$, we do not adopt this inessential assumption. Our functional form of the function $C\left(R_{t}\right)$ is different from $\hat{C}(q)$. Following basic assumptions, Eqs. $(1,2,3,4)$, are essentially the same as Rowthorn. Hereafter, we refer to the social cost per unit time as 'intervention cost' in the form of $C\left(R_{t}\right)$. The following are assumed in the function $C\left(R_{t}\right)$.

The condition without intervention measures corresponds to $R_{t}=R_{N}$, in which $C\left(R_{N}\right)=$ 0 . The cost should increase as the effective reproduction number decreases. The rate of increase of $C\left(R_{t}\right)$ should also increase as the effective reproduction number decreases. This is because society can take cost-effective measures, such as handwashing, to achieve a small decrease in $R_{t}$. If society must further decrease $R_{t}$, it must take costlier measures. Thus, we can set the following conditions on the intervention cost function $C\left(R_{t}\right)\left(0<R_{t} \leq R_{N}\right)$, where an example is shown in Figure 1.

$$
\begin{gathered}
C\left(R_{t}\right) \text { is twice continuously differentiable, } \\
C\left(R_{N}\right)=0 \\
\frac{d C\left(R_{t}\right)}{d R_{t}} \leq 0 \\
\frac{d^{2} C\left(R_{t}\right)}{d R_{t}^{2}} \geq 0
\end{gathered}
$$


The measure taken by spending the intervention cost, $C(R)$, is to decrease infected population (the number of infected persons who are capable of transmitting infections), $I$. The more the infected population decreases for fixed intervention costs, the more society benefits from the measure. The 'benefit of a decrease in the infected population' is evaluated as the 'decrease in the cost of the infected population'. We set this 'infection cost' $M$ to be proportional to the infected population, $I$, which includes medical costs and infected patients' incurred losses. This yields

$$
M(t)=c_{1} I(t),
$$

where $c_{1}$ is a constant. This assumption is also the same as Rowthorn ${ }^{2)}$. The total cost per unit of time is the sum of the intervention cost and the infection cost, namely $C(t)+M(t)$. The optimisation issue is to find $R(t)$, which minimises the integrated total cost over a certain period,

$$
\int[C(t)+M(t)] d t,
$$

In other words, this will find $R(t)$ that minimises the average of the total cost over a certain period.

To find the optimised intervention process specified by a protocol of $R(t)$ for a targeted period, we must consider the dynamics of the infected population. Here, we begin with the SIR model proposed by Kermack and McKendrick ${ }^{9)}$ because most of previous studies, including Routhorn et al., have assumed that it is the simplest fundamental model that describes the basic dynamics of epidemics. It models the exponential growth of the infected population in the outbreak stage, the peak of the infected population, and transitioning to the end stage ${ }^{13)}$. However, it should be noticed that the following results are not restricted to the SIR framework, as will be described later. 


\section{Pandemic Dynamics}

We start with the SIR model for pandemic dynamics for its simplicity and popularity. The model comprises a set of differential equations that describes the epidemic disease propagation, in which the population is divided into three states: $S(t)$, the population ratio of susceptible persons, $I(t)$, the ratio of infected persons, and $\hat{R}_{\text {rec }}(t)$, the ratio of those who have recovered (or died). This formulation considers a closed population that is conserved. Note that we use the notation $\hat{R}_{\text {rec }}$ for recovered persons, instead of the conventional notation, $R$, because we use $R_{t}$ for the average reproduction number.

$$
\begin{gathered}
\frac{d S(t)}{d t}=-\beta S(t) I(t), \\
\frac{d I(t)}{d t}=\beta S(t) I(t)-\gamma I(t), \\
\frac{d \hat{R}_{\mathrm{rec}}(t)}{d t}=\gamma I(t),
\end{gathered}
$$

where $\beta$ and $\gamma$ are the infection and recovery rates, respectively. The sum of the three population ratios remains constant:

$$
S(t)+I(t)+\hat{R}_{\mathrm{rec}}(t)=1 .
$$

Because of this conservation law, the number of independent variables in the model is two.

In the following, we evaluate the infected population, $I(t)$. Equation (??) leads to

$$
\frac{d I(t)}{d t}=\gamma\left[\frac{\beta S(t)}{\gamma}-1\right] I(t) .
$$

We restrict ourselves to the period before the vicinity of the infection peak, because this period 
is the most important and universal characteristic of pandemics, as will be discussed later. In this period, $S(t)$ is replaced by $S(0)$. This approximation is accurate in major parts of the first outbreak and its recurrent phases ${ }^{14)}$, as shown in the figure 1 in the Extended Data. Because of this approximation, the number of independent variables of this model is reduced to one. Then, Eq. (11) leads,

$$
\frac{d I(t)}{d t}=\gamma\left[\frac{\beta S(0)}{\gamma}-1\right] I(t) .
$$

We restrict ourselves to $\gamma$ being fixed, like Rowthorn ${ }^{2)}$. If the set of parameters $\frac{\beta S(0)}{\gamma}>1$, the infections start spreading in Eq. (12) ${ }^{15)}$. The change in $\beta$ in $\frac{\beta S(0)}{\gamma}$ changes the dynamics of the pandemic. The set of parameters is the effective reproduction number:

$$
R_{t}=\frac{\beta S(0)}{\gamma}
$$

where $R_{t}$ corresponds to the basic reproduction number, $R_{0}$, if the following two assumptions are satisfied: 1) $\beta$ has an uncontrolled value and 2) $S(0)=1$. The infected population increases when $R_{t}>1$ and decreases for $R_{t}<1$.

Equation 12 becomes, with $\Delta_{R}=R_{t}-1(>0)$,

$$
\frac{d I(t)}{d t}=\gamma \Delta_{R} I(t)
$$

At $R_{t}=1$, the infected population is stationary, as $\Delta_{R}=0$. The infection-spreading phase of pandemics generally obeys exponential dynamics characterised by the effective reproduction number, except for the vicinity of the infection peak. Thus the following results are not restricted to specific modelling. In this formulation, the infected population, $I(t)$, is the only variable that describes the state of the system. In the following sections, we will show the universal properties of the system by analysing the cyclic process of the state variable $I(t)$. 


\section{Irreversible cost in on/off-type intervention process}

Next, we evaluate the costs of on/off-type infection control (see Figure 2) and compare it with the costs of keeping the infected population stationary, where we assume that both processes have the same average effective reproduction number, $\left\langle R_{t}\right\rangle=1$. The present on/off-type intervention forms a cycle of both $R_{t}$ and $I(t)$, as shown in the following, in which a set of lockdown and recurrence is the extreme example. We set the amplitude of the cycle in the effective reproduction number around $R_{t}=1$ as ' $\Delta$ ', where $\Delta=\left|R_{t}-1\right|$. The cyclic process (with time interval $T$ ) is as follows:

Stage 1) $0<t<T: I_{0} \rightarrow I_{1}\left(>I_{0}\right)$ with $R_{t}=1+\Delta$,

Stage 2) $T<t<2 T: I_{1} \rightarrow I_{0}$ with $R_{t}=1-\Delta$,

Stage 3) $2 T<t<3 T: I_{0} \rightarrow I_{3}\left(<I_{0}\right)$ with $R_{t}=1-\Delta$,

Stage 4) $3 T<t<4 T: I_{3} \rightarrow I_{0}$ with $R_{t}=1+\Delta$.

By integrating Eq. (14) from $t=0$ to $T$ with $R_{t}=1+\Delta$, we obtain the infected population $I$ at the end of Stage 1,

$$
I(T)=I_{0} e^{\gamma T \Delta}
$$

Similarly, replacing $\Delta_{R}$ in Eq. (14) by ' $-\Delta$ ' and using Eq. (15), we obtain $I(2 T)$ at the end of Stage 2:

$$
I(2 T)=I_{0}
$$

Stages 3 and 4 also yield

$$
I(4 T)=I_{0}
$$


We have confirmed that Stages 1 through 4 form a typical cyclic process of the state variable, $I(t)$, around a stationary state kept by $R_{t}=1$, where the infected population returns to its original value.

We calculated the average infected population to evaluate the infection cost in the cycle. Using Eqs. (14) and (15), we have, for Stages 1 and 2,

$$
\int_{0}^{T} I_{\text {Stage } 1}(t) d t+\int_{T}^{2 T} I_{\text {Stage } 2}(t) d t=I_{0}\left[\int_{0}^{T} e^{\gamma \Delta t} d t+\int_{T}^{2 T} e^{\gamma \Delta T} e^{-\gamma \Delta(t-T)} d t\right]=I_{0} \int_{0}^{T}\left[e^{\gamma \Delta t}+e^{\gamma \Delta(T-t)}\right] d t
$$

Similarly, for Stages 3 and 4, we have

$$
\int_{2 T}^{3 T} I_{\text {Stage3 }}(t) d t+\int_{3 T}^{4 T} I_{\text {Stage } 4}(t) d t=I_{0} \int_{0}^{T}\left[e^{-\gamma \Delta t}+e^{\gamma \Delta(t-T)}\right] d t
$$

Thus, we obtain,

$$
\frac{1}{4 T} \int_{0}^{4 T} I(t) d t=\frac{I_{0}}{\gamma \Delta T} \sinh (\gamma \Delta T)=I_{0}+\frac{I_{0}(\gamma \Delta T)^{2}}{3 !}+O\left((\gamma \Delta T)^{4}\right) .
$$

The stationary infected population at $R_{t}=1$ during the same period, $4 T$, is $I_{0}$. This proves that the average infected population in this cycle is always higher than that of the stationary state. This result yields directly through Eq.(5) :

$$
\langle M\rangle_{\text {cycle }}>\langle M\rangle_{R_{t}=1}
$$

where $\langle M\rangle$ denotes the time-average of $M$. Thus, the average infection cost for this cycle is higher than that of the stationary state. Figure 3 shows how the average infection cost depends on the amplitude of the cycle $\Delta$.

Next, we calculate the average intervention cost during the cycle. The average intervention 
cost, weighing the two effective reproduction numbers, $R_{t}=1+\Delta$ and $R_{t}=1-\Delta$ equally $(\Delta>0)$ for the same period is

$$
\left\langle C\left(R_{t}\right)\right\rangle_{\text {cycle }}=\frac{C(1+\Delta)+C(1-\Delta)}{2} .
$$

The cost $C(1+\Delta)$ is evaluated as follows:

$$
C(1+\Delta)=C(1)+\int_{1}^{1+\Delta} \frac{d C\left(R_{t}\right)}{d R_{t}} d R_{t}
$$

From Eq. (4) we find

$$
\frac{d C\left(R_{t}\right)}{d R_{t}}>\left.\frac{d C\left(R_{t}\right)}{d R_{t}}\right|_{R_{t}=1}\left(\text { for } 1<R_{t} \leq R_{N}\right)
$$

Then, we have

$$
C(1+\Delta)>C(1)+\left.\frac{d C\left(R_{t}\right)}{d R_{t}}\right|_{R_{t}=1} \Delta .
$$

169 Since $\frac{d C\left(R_{t}\right)}{d R_{t}}<\left.\frac{d C\left(R_{t}\right)}{d R_{t}}\right|_{R_{t}=1}$ for $0<R_{t}<1$,

$$
C(1-\Delta)>C(1)-\left.\frac{d C\left(R_{t}\right)}{d R_{t}}\right|_{R_{t}=1} \Delta
$$

We obtain through Eqs. (25) and (26)

$$
\left\langle C\left(R_{t}\right)\right\rangle_{\mathrm{cycle}}=\frac{C(1+\Delta)+C(1-\Delta)}{2}>C(1),
$$

in which $C(1)$ equals the intervention cost in a stationary state with $R_{t}=1$. Thus, we find that the average intervention cost, $\left\langle C\left(R_{t}\right)\right\rangle$, is also higher in this cycle than keeping a stationary state with $R_{t}=1$. Figure 4 illustrates how the intervention cost depends on the amplitude of 
the cycle $\Delta$, where we use the model in Figure 1.

The results show that the cycle of infection control around the stationary state provokes a higher average infected population, $\langle I(t)\rangle$, and also a higher intervention cost compared to the stationary state. Because the variable of the state, $I(t)$, finally returns to the initial state in the cycle, the cycle above results in a waste of social resource compared to a stationary state. The economic irreversibility that society cannot retrieve the dissipated social resource is similar to entropy production (or free energy decreases) in thermodynamics ${ }^{16)}$.

The total cost, $C\left(R_{t}\right)+M(t)$, for the cycle thus satisfies the inequality

Average of the total cost of the cyclic process $>$ That of the stationary process

even if the two processes have the same average effective reproduction number $\left\langle R_{t}\right\rangle=1$.

We have learned that society cannot produce extra benefits (decrease of infected population) in the cyclic process compared to keeping the infected population constant while it pays extra intervention costs in the cycle. In addition to this, society also incurs the demerit (increase of infected population) in the cycle. Note that this inequality holds irrespective of specific parameters, which conflicts previous studies on the economic efficiency of infection control. This inequality clearly illustrates how on/off-type infection control against pandemics costs society.

\section{Irreversible Cost for Delaying Measures}

Now, we will show the implication of economic irreversibility by the effect of delaying measures against pandemics. We compare the two processes having the same initial and final states, $I_{0}$. Only the intermediate states are different between the two processes. 
Process 1) Do not perform infection control initially or perform small intervention at $t=0$ with $R_{t}=R_{a}$, in which $1<R_{a} \leq R_{N}$, until some critical time ( $\left.t=t_{a}\right)$ just before serious problems such as the crash of medical capacity arise. Then, infection control is performed at $t=t_{a}$ to achieve a constant $R_{t}<1$ to decrease $I(t)$ back to $I_{0}$. This process is similar to the combined process of Stages 1 and 2 in Figure 2. However, the choice of $R(t)$ before and after $t=t_{a}$ is arbitrary.

Process 2) Perform infection control to achieve $R_{t}=1$ immediately at $t=0$.

Here, we assume $R_{N}>1$ for both processes.

The advantage of Process 1 is that there is no or small intervention cost, $C\left(R_{a}\right)<C(1)$, between $t=0$ and $t=t_{a}$. Compared with the decision to initially take measure $R_{t}=1$ (Process 2), this saves intervention costs between $t=0$ and $t_{a}$ :

$$
\int_{0}^{t_{a}}\left[C(1)-C\left(R_{a}\right)\right] d t
$$

Thus, it is the matter whether the saving of the intervention cost (Eq. (29) at $t=t_{a}$ ) remains positive even at the final stage, $t=t_{b}$, when the state returns to its initial state, $I_{0}$. Thus, we calculate the average intervention cost of Process $1,\left\langle C\left(R_{t}\right)\right\rangle_{\text {delay }}$, during the period from $t=0$ to $t=b$. From Eq. (14), the state of $I(t)$ at $t=t_{a}$ is $I\left(t_{a}\right)=I_{0} e^{\gamma t_{a} \Delta_{a}}$, where $\Delta_{a}=R_{a}-1$. We assume that $I(t)$ returns to $I_{0}$ at $t=t_{a}+t_{b}$, and $R_{t}=R_{b}=1-\Delta_{b}\left(0<\Delta_{b}<1\right)$ for $t_{a}<t \leq t_{b}$. Then, we have $I\left(t_{a}+t_{b}\right)=I\left(t_{a}\right) e^{-\gamma t_{b} \Delta_{b}}$. As $I\left(t_{a}+t_{b}\right)=I_{0}$, we obtained the equality

$$
t_{a} \Delta_{a}=t_{b} \Delta_{b}
$$


Then, the average intervention cost between $t=0$ and $t=t_{b}$ is written as

$$
\left\langle C\left(R_{t}\right)\right\rangle_{\text {delay }}=\frac{t_{a}}{t_{a}+t_{b}} C\left(1+\Delta_{a}\right)+\frac{t_{b}}{t_{a}+t_{b}} C\left(1-\Delta_{b}\right) .
$$

From Eqs. (25) and (26), Eq. (31) satisfies the following condition:

$$
\left\langle C\left(R_{t}\right)\right\rangle_{\text {delay }}>\frac{t_{a}}{t_{a}+t_{b}}\left[C(1)+\left.\frac{d C}{d R_{t}}\right|_{R_{t}=1} \Delta_{a}\right]+\frac{t_{b}}{t_{a}+t_{b}}\left[C(1)-\left.\frac{d C}{d R_{t}}\right|_{R_{t}=1} \Delta_{b}\right]
$$

Using Eq. (30), the right-hand side of Eq. (32) equals C(1). Thus, we obtain

$$
\left\langle C\left(R_{t}\right)\right\rangle_{\text {delay }}>C(1)
$$

The right-hand side is the average intervention cost of Process 2. The average intervention cost, $\left\langle C\left(R_{t}\right)\right\rangle_{\text {delay }}$ in the delaying measure is found to be higher than that for a stationary infection state. The inequality has universality because Eq. (33) holds for any process with linear functions with parameters $\Delta_{a}$ and $\Delta_{b}$. Furthermore, because any integrable function can be decomposed into a set of linear functions with arbitrary precision, Eq. (33) holds for any process of integrable $R(t)$ on the condition that the variable of state $I(t)$ returns to its initial state.

Apparently, the infection cost satisfies the similar inequality as above,

$$
\left\langle M\left(I\left(R_{t}\right)\right)\right\rangle_{\text {delay }}>M(I(1)),
$$

as the average infected population is higher in the delaying measure than in a stationary infection population with $R_{t}=1$. The results show that society with a delaying measure must pay more intervention and infection costs during the process until the state $I(t)$ returns to its original state, even if it temporarily saves intervention costs. In other words, once the infected population 
increases, society cannot return to the previous lower infection state without paying extra costs instead of keeping a stationary state, as in Process 2. An increase in the infected population always results in economic irreversibility in pandemics, except for the vicinity of the infection peak. The universal result of the model is again independent of the details of the system.

\section{Discussion and Conclusion}

This study theoretically analysed the fundamental structure of economic irreversibility in infection control process during the infection-spreading phase. Delaying measures against the spread of infection results in cost increases. Once the state variable $I(t)$ is increased, the system is irreversible because it cannot return to the previous low-infection state without extra expenditures compared to keeping the stationary state of the low-infection. These general results contradict the naive idea that infection control always results in economic damage.

The merit of keeping the infection population constant has been previously discussed by Rowthorn ${ }^{2)}$; Rowthorn stated, 'The most robust conclusion is that, if a relatively inexpensive way can be found to reduce the net reproduction ratio to $r=1$, that is, the policy to aim for in the medium term'. This numerical finding is consistent with our analytical result. It should be noted that the present result cannot show the level to which the society should decrease the infected population. Additionally, our analysis is restricted to a principal part of the pandemic, namely, the infection-spreading phase. These are limitations of our study.

The validity of the present study is subject to assumptions of the methodology. In addition to the conventional methodological assumptions of a homogeneous mixing of the infected and susceptible populations ${ }^{17)}$ and constant rates ${ }^{9)}$, we used the two principal assumptions:

1. The intervention cost depends on the effective reproduction number, $R_{t}$, and its cost function $C\left(R_{t}\right)$ is concave up as in Eq.(4). 
2. Without infection control, the epidemic is in the infection-spreading phase, and increases and decreases in the infected population obey exponential dynamics as in Eq. (14).

The first assumption is the same as the assumption of previous research ${ }^{2,4)}$ through the relation $R_{t}=R_{N}(1-q(t))$, which is intuitively understandable as shown in the section "Formulation with Cost-Benefit Analysis." The exponential dynamics in the second assumption is a common feature of pandemics. Thus, the results are not restricted to the specific modelling but are general features in most pandemics, as long as the infection-spreading phase is expected to last longer than the period of variation of the infected population.

Our study does not offer concrete cost values like conventional cost-benefit analyses. However, the present result reveals the universal structure of the costs, which is independent of the coefficients of the cost, for example, $c_{1}$, of Eq. (5). The universality found in this study is similar to thermodynamics in physics ${ }^{18)}$. The theory of thermodynamics alone does not reveal the physical quantity of a system as in the present study. However, it provides a quantitative relationship among physical variables and shows physical irreversibility. Physical irreversibility is similar to the present result that an increase in the infected population is economically irreversible.

Irreversibility of thermodynamics is caused by the deviation from thermal equilibrium. Carnot's cycle is known as a reversible thermodynamics process, which converts thermal energy into mechanical energy at maximum efficiency ${ }^{18)}$. This is called Carnot's efficiency and is analogous to $\mathrm{CBA}$ in the sense that $\mathrm{CBA}$ evaluates the efficiency of conversion from social intervention cost into benefit (decrease in infected population, in the present case). Optimal energy conversion is available in Carnot's cycle because the cycle is at equilibrium, and thus, there is no entropy production. In a non-equilibrium stationary state, it requires a finite cost to keep the system stationary ${ }^{19,20)}$, in which the efficiency of energy conversion is different from that at equilibrium. However, even if the system is out of equilibrium, the efficiency of energy 
conversion $^{21)}$ and an equality on irreversibly work ${ }^{22)}$ can be analytically discussed with thermodynamics and statistical mechanics. The present system corresponds to non-equilibrium, even in the stationary state of a constant infected population, because the stationarity is maintained by spending the infection control cost with $C\left(R_{t}=1\right)>0$ to inhibit an increase in the infected population. Therefore, the application of concepts and methodology of non-equilibrium thermodynamics into CBA would be interesting ${ }^{23)}$ because economic irreversibility ${ }^{24,25,26)}$ exists and has universality, as shown here.

Our analysis of the infection-spreading phase explicitly showed that the increased state is economically irreversible once the infected population increases, which is the universal result. This result is not only applicable to COVID-19 and whether or not 'herd immunity' exists ${ }^{11,12)}$. To the best of our knowledge, this is the first analytical study of economic efficiency in pandemic control. The result may provide guiding principles for infection control in pandemics as thermodynamics gives several guiding principles for nature and industries. The following question has not yet been clarified by our model: 'To which level we should decrease infected population?' The question includes whether we should aim at the eradication of infection. Analytical studies to find conditions that determine the most effective infection control are important and challenging for the future.

\section{References}

[1] Bloom, D.E., \& Canning, D. Epidemics and Economics, PGDA Working Paper No. 9 http://www.hsph.harvard.edu/pgda/working.htm, Sch. of Pub. Health, Harvard University (2006).

[2] Rowthorn, R. A cost-benefit analysis of the COVID-19 disease Covid Economics, Issue 9, 97-106 (2020). 
[3] For example, articles at Covid Economics, Centre for Economic Policy Research (Vetted and Real-Time Papers): https://cepr.org/content/covid-economics-vetted-and-real-timepapers-0 (2020).

[4] Rowthorn, R. \& Maciejowski, J.A. Cost-benefit analysis of the Covid-19 disease, Oxford Rev. Econ. Policy, https://doi.org/10.17863/CAM.54369 (2020).

[5] Eichenbaum, M.S., Rebelo, S. \& Trabandt, M. The Macroeconomics of Epidemics, Preprint at https://www.kellogg.northwestern.edu/faculty/rebelo/htm/epidemics.pdf (2020).

[6] Acemoglu, D., Chernozhokov, V., Werning, I., Whinston, M.D. Working Paper 27102, (National Bureau of Economic Research, 2020).

[7] Boardman, A.E., Greenberg, D.H., Vining, A.R., \& Weimer, D.L. Cost-benefit analysis: concept and practice (2nd Ed.) (Prentice Hall, 2001).

[8] Nas, T. F. Cost-Benefit Analysis: Theory and Application, (Sage Publication, 1996).

[9] Kermack, W.O. \& McKendrick, A.G. A contribution to the mathematical theory of epidemics, Proc. Royal Soc. A, 115, 700-721 (1927).

[10] NHK News, Chief Cabinet Secretary asked governors to keep hospitalization facilities for infected citizens with mild symptoms, Japan Broadcasting Corporation, July 28 (in Japanese), https://www.nhk.or.jp/politics/articles/statement/42463.html (2020).

[11] To, K. K-W., Hung, I.F-N. et al. COVID-19 re-infection by a phylogenetically distinct SARS-coronavirus-2 strain confirmed by whole genome sequencing, Clin. Infect. Dis., https://doi.org/10.1093/cid/ciaa1275 (2020). 
[12] Tillett, R., Sevinsky, J. et al. Genomic evidence for a case of reinfection with SARS-CoV2, SSRN, https://dx.doi.org/10.2139/ssrn.3680955 (2020).

[13] Martcheva, M. An Introduction to Mathematical Epidemiology (Springer-Verlag 2010).

[14] Vynnycky, E. \& White, R.G. An Introduction to Infectious Disease Modeling (Oxford University Press, 2010).

[15] Earn, D.J.D. A light introduction to modelling recurrent epidemics, in Mathematical Epidemiology (eds F. Brauer, P. Driessche, J. Wu) 3-17 (Springer-Verlag, 2008).

[16] Nicolis, G. \& Prigogine, I. Exploring Complexity (R. Piper GMbH \& Co., 1989).

[17] Giesecke, J. Modern Infection Disease Epidemiology (3rd Ed.), (Taylor \& Francis, 2017)

[18] Callen, H. Thermodynamics (John Wiley \& Sons, 1960).

[19] Hondou, T. \& Sekimoto, K. Unattainability of Carnot efficiency in Brownian heat engine, Phys. Rev. E, 62, 6021-6025 (2000).

[20] Hondou, T. \& Takagi, F. Irreversible operation in a Stalled state of Feynman's ratchet, J. Phys. Soc. Jpn., 67, 2974-2976 (1998).

[21] Kamegawa, H. \& Hondou, T. \& Takagi, F. Energetics of a forced thermal ratchet, Phys. Rev. Lett., 80, 5251-5254 (1998).

[22] Jarzynski, C. Nonequilibrium equality for free energy differences, Phys. Rev. Lett., 78, 2690-2693 (1997).

[23] Tsirlin, A.M. Irreversible microeconomics:Optimal processes and control, Autom. Remote. Control., 62, 820-830 (2001). 
[24] Martinas K. About Irreversibility in Economics, Open Sys. Inf. Dyn., 7, 329-364 (2000).

[25] Amir, S. The role of thermodynamics in the study of economic and ecological systems, Ecol. Econ., 10, 125-142 (1994).

[26] Ayres, R.U. \& Nair I. Thermodynamics and economics, Phys. Today, 37, 62-71 (1984).

\section{Data Availability}

Data sharing is not applicable to this article because no datasets were generated or analysed during the current study. All figures except for the lower curve in Extended Figure 1 are generated by the explicit solutions shown in the text. The lower curve in Extended Figure 1 is generated by numerical simulation (Euler method). As the initial condition is fully described in the extended figure legend, the figure is also reproducible.

\section{End notes}

\section{Acknowledgements}

The author acknowledges T. Onai, S. Yonemura, K. Hirata, and S. Munakata for their fruitful discussions. S. Takagi, R. Seto, M. Arikawa, M. Sano, H. Chaté and M. Wallis are also acknowledged by the author for their critical reading of the manuscript and helpful comments. We would like to thank Editage for English language editing. This work was supported by the Japanese Grants-in-Aid JSPS Nos. 16H01820 and 20H00002.

\section{Authors contributions}

T.H. solely contributed to this study. 


\section{${ }_{357}$ ORCID ID}

358 https://orcid.org/0000-0001-5520-6688 (T.H.)

${ }_{359}$ Competing interests

360 The author has no completing interest.

${ }_{361}$ Corresponding author

362 Tsuyoshi Hondou, hondou@ mail.sci.tohoku.ac.jp 
${ }_{363}$ Figures

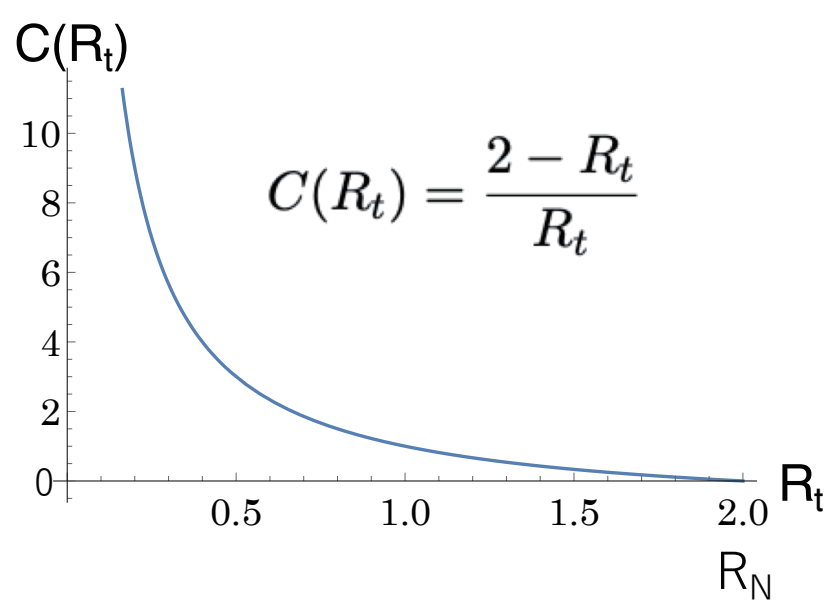

Figure 1: An example of intervention cost, $C$.

365

Here $C\left(R_{t}\right)=\left(2-R_{t}\right) / R_{t}$, where $R_{N}=2$. 


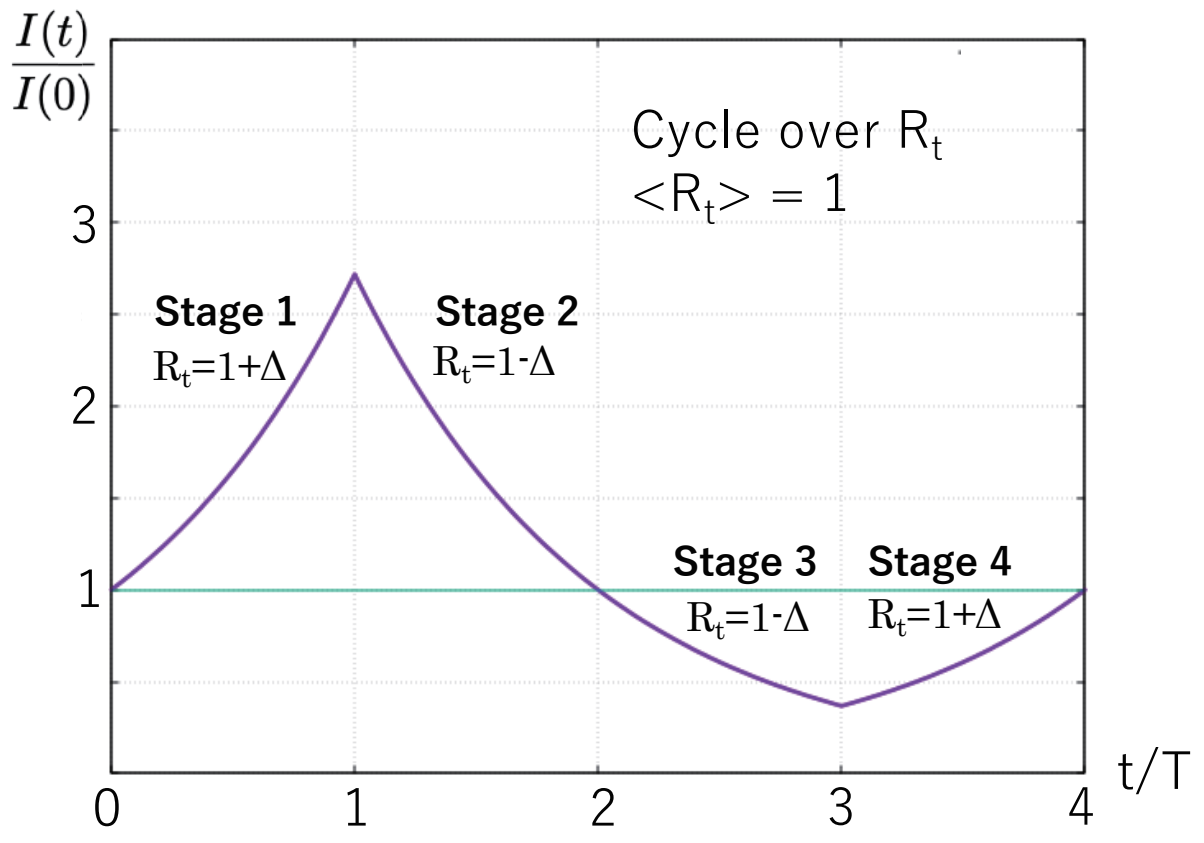

Figure 2: Trace of infected population during the cyclic process of infection control.

It is shown that the infected population is also cyclic, and returns to the initial state at the end of the cycle. The average infected population $\langle I(t)\rangle$ over the cycle is larger than that for keeping the infected population stationary. 


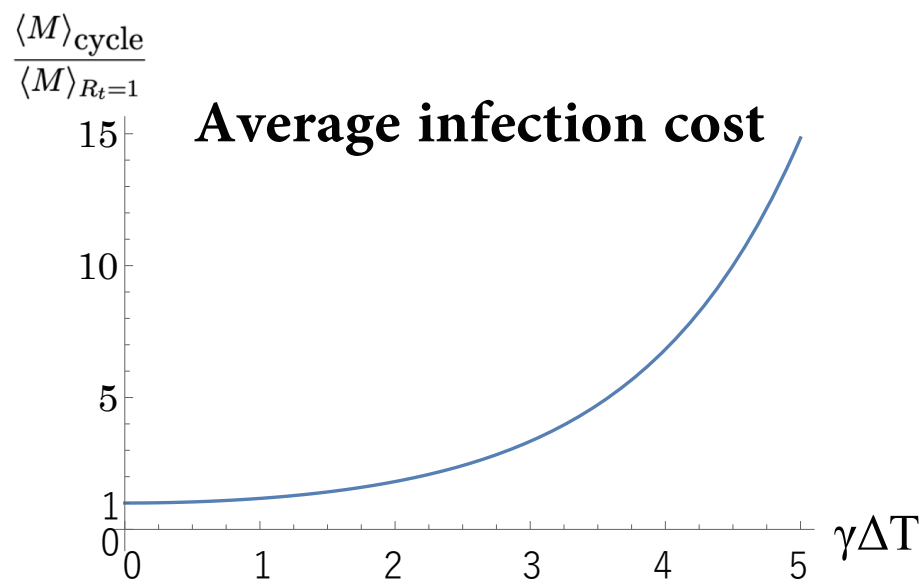

Figure 3: Large oscillation of intervention results in large infection cost.

The average infection cost, $\langle M(I(t))\rangle$, increases monotonically and exponentially as the amplitude of $R_{t}$ in the cycle, $\Delta$, increases. The vertical axis is normalised by the average infection cost for the stationary state with $R_{t}=1$, having an average effective reproduction number equal to that of the cycle. As the state variable $I(t)$ returns to its initial state in the cycle, the increase in average infection cost is irreversible. 


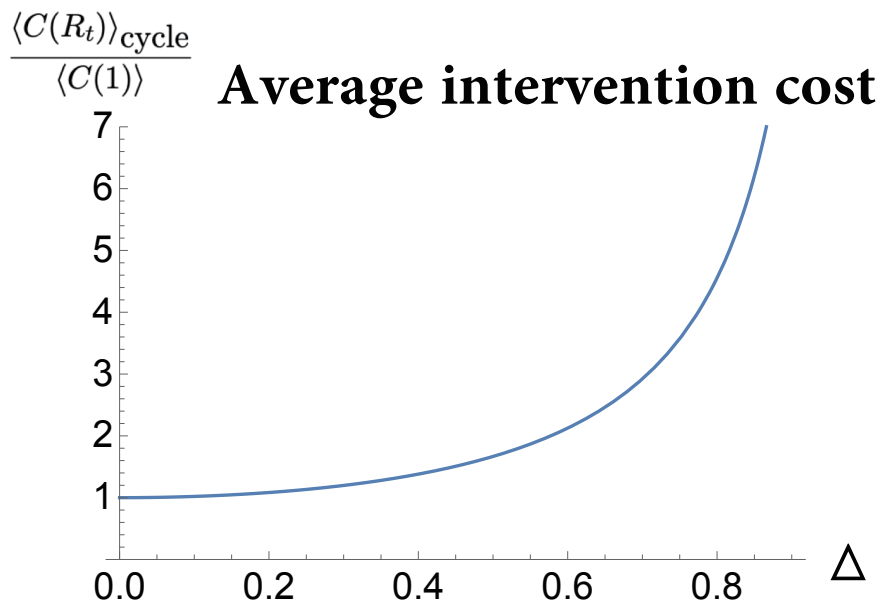

Figure 4: Large oscillation of intervention also results in large intervention cost.

The average intervention cost, $\left\langle C\left(R_{t}\right)\right\rangle$, increases exponentially as the amplitude of $R_{t}$ in the cycle, $\Delta$, increases. The vertical axis is normalised by the average intervention cost for the stationary state with $R_{t}=1$, having an average effective reproduction number equal to that of the cycle. We use $R_{N}=2$ and $C\left(R_{t}\right)$ of Figure 1 . The increase in average intervention cost in the cycle does not contribute to the benefit (decrease in average infection cost) at all, as Figure 3 shows. 


\section{Extended Data}

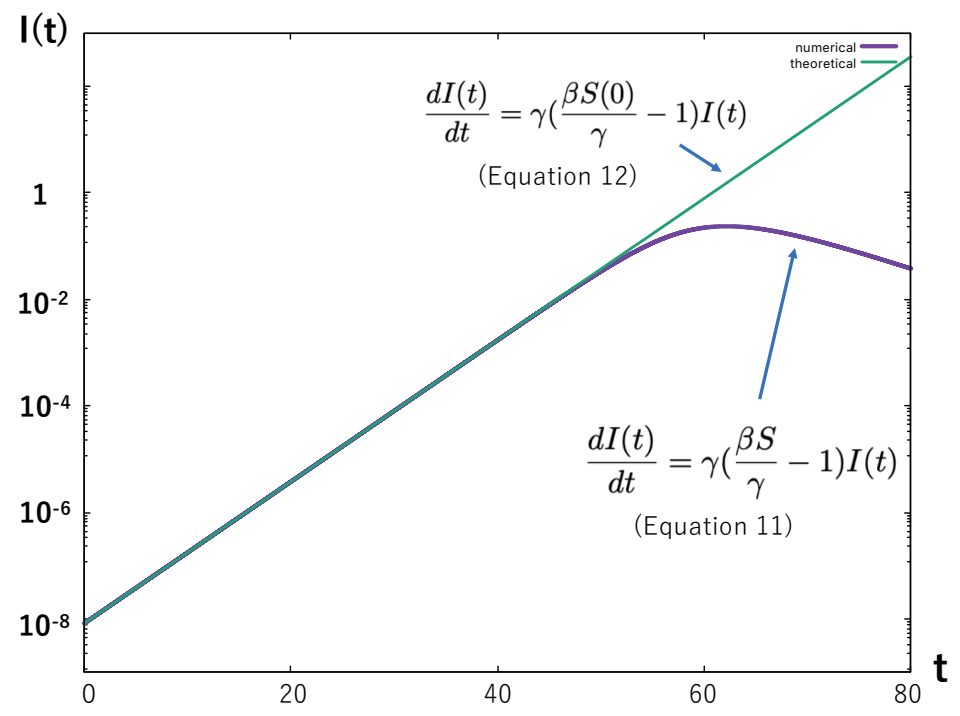

\section{Extended Figure 1:}

The validity of theoretical approximation leading to Equation 12 is shown.

We replaced the variable of susceptive persons, $S(t)$, in Eq. (11) with respect to the initial value $S(0)$ (Eq.(12)) for the evaluation of the infected population in the infection-spreading phase because $S(t)$ is a slow variable. The upper line is a solution of Equation 12. The lower line is a solution of Equation 11 obtained using numerical calculation. The figure illustrates that the approximation over $S(t)$ is precise in the infection-spreading phase, except for the vicinity of the infection peak. Here, we used $\beta=0.51$ and $\gamma=0.204$, which corresponds to the basic reproduction number $R_{0}=2.5$ for demonstration. Numerical calculation for Equation 11 is performed using the Euler method, in which the initial values are as follows: Total population $N=1.2 \times 10^{8}+1, S(0)=1.2 \times 10^{8} / N, I(0)=1 / N, \hat{R}_{\mathrm{rec}}(0)=0 / N$. 